\title{
Airway involvement in pulmonary tuberculosis
}

\author{
Pierre Goussard, Robert Gie
}

Airway involvement is relatively commonly seen in children with primary tuberculosis (TB), but in only a small group of children is the compression severe, needing intervention. The incidence of children with airway obstruction due to primary $\mathrm{TB}$ in the chemotherapeutic era is not known. In two recent reports the incidence of complicated lymph node disease varied from $8 \%$ to $38 \%$ in children less than 15 years of age. ${ }^{1,2}$

The trachea and the two main bronchi are the airways most often affected by the enlarged glands. Upper-airway involvement is rare in children; most reports of upper-airway TB have been in adults. However, two forms of upperairway TB have been described in children: laryngeal TB and retropharyngeal TB abscess.

In children other conditions also cause airway compression and it is important to include these in the differential diagnosis. External airway compression can be caused by congenital malformations, vascular compression and enlarged mediastinal lymph nodes. Airway compression can also be confused with airway obstruction, with foreign-body inhalation a common cause of intraluminal obstruction.

\section{Clinical presentation}

Children with airway compression present with a wide variation of symptoms and signs. The clinical presentation depends on a number of factors:

1. Age of the child. Younger children with relatively smaller airways have the largest risk of being symptomatic. Their airway obstruction, which can be quite severe, is often precipitated by an acute viral infection with the viral-induced inflammation causing additional obstruction.

2. Anatomical site of the involved airway. The involvement can be supraglottic, extrathoracic, intrathoracic, or combinations of these. The most severe forms of obstruction are seen when both the trachea and one or both of the main bronchi are involved. Extrathoracic airway obstruction presents with stridor, which may be present during inspiration or both inspiration and expiration. Intrathoracic obstruction presents with monophonic wheezing.

3. The degree of luminal obstruction. TB lymph gland enlargement involves the airways in various pathological ways.

Department of Paediatrics and Child Health, Desmond Tutu Tuberculosis Centre, Stellenbosch University, Tygerberg, W Cape

Pierre Goussard, MMed (Paed)

Robert Gie, FCP (Paed)

Corresponding author: P Goussard (pgouss@sun.ac.za)
The enlarged glands may only cause external compression of the airways. Further involvement leads to herniation of the glands into the airways causing worsening obstruction but rarely complete obstruction of the airway. The degree of obstruction leads to different clinical presentations. Airway compression causes wheezing audible on one or both sides of the chest depending on the degree of obstruction. These children are misdiagnosed as asthmatics but respond poorly to asthma treatment. Children with partial obstruction of the airway may develop a ball-valve effect, where air can enter the lung but is trapped on expiration. There is hyperinflation and hyper-resonance of the affected side of the chest, the breath sounds over the involved lung are decreased, and prolonged expiration is audible. When the obstruction is complete, lung or lobar collapse can develop with reduced or no ventilation to the affected part of the lung. Bronchus intermedius is the most vulnerable part of the airway, and collapse of the right middle and lower lobes may occur. Airway compression is common in children with expansile pneumonia caused by Mycobacterium tuberculosis (MTB). ${ }^{3}$

4. Other factors. Airway obstruction occurs in both HIVinfected and HIV-uninfected children. The size of the lymph nodes may increase during anti-TB treatment and airway obstruction may worsen. This also occurs in children receiving both TB and antiretroviral drugs. ${ }^{4}$ Luminal TB is rare in children and is mostly seen in adults with TB. In these cases there is no compression of the airway but involvement of the luminal wall.

\section{Diagnosis of airway compression}

Most severe forms of airway compression can be diagnosed from plain chest X-rays (CXRs) and further investigation is only necessary in complicated cases.

Four patterns of airway obstruction can be recognised on CXR: (i) airway narrowing; (ii) ball-valve effect; (iii) expansile pneumonia; and (iv) lobar collapse.

\section{Airway narrowing}

The most important groups of lymph nodes causing airway compression are the paratracheal, hilar and subcarinal lymph nodes. Lymph gland obstruction of the airways is commonly clearly visible on a CXR. Paratracheal lymph glands can cause tracheal compression. Any deviation of the trachea to the left is abnormal, except when a right-sided aortic arch is present, and can indicate paratracheal lymph gland compression of the trachea. Subcarinal glands may also be difficult to detect, but if both main bronchi are narrowed, enlarged subcarinal lymph nodes may be present. Shifting of the para-oesophageal 
adhesion line may also indicate enlarged subcarinal lymph nodes.

The frontal high kilovolt $(\mathrm{kV})$ radiograph may be useful to assess the effect of enlarged TB lymph glands on the tracheobronchial tree. It has been demonstrated that the specificity for the detection of TB lymph nodes increases from $74.4 \%$ to $86.6 \%$ with the addition of high- $\mathrm{kV}$ radiographs. ${ }^{5}$

\section{Ball-valve effect}

This is seen on a CXR as unilateral hyperinflation because of the fact that air is trapped by the 'check valve' effect of an incompletely obstructed airway. The involved lung or lobe is enlarged, has reduced vascularity and can herniate across the midline if severe air trapping is present.

\section{Expansile pneumonia}

Expansile pneumonia caused by MTB affects mostly the upper lobes. Expansile pneumonia is characterised by the dense opacification of the enlarged lobe with displacement of the fissures. Air bronchograms are usually not visible. Airway compression is seen in $92 \%$ of cases on plain CXR.

\section{Lobar collapse}

Lobar collapse is uncommon with the right middle and lower lobes being most commonly involved.

\section{Management of airway obstruction}

Children with airway obstruction caused by MTB are treated with the standard 3-drug regimen to which corticosteroids (prednisone $2 \mathrm{mg} / \mathrm{kg} / \mathrm{d}$ ) are added for the first month of treatment. If there is clinical and radiological improvement, the treatment is continued and the corticosteroids are weaned over the next month. There is very little information on the use of corticosteroids in the treatment of TB glandular obstruction of the airways. Early reports from the 1960s were that corticosteroids were effective in decreasing airway obstruction if given early in the course of the disease. These claims were not supported in a double-blind study looking at the effect of prednisone on lymph node disease. ${ }^{6}$ In a follow-up study the group receiving prednisone had an improved outcome when compared with the placebo group (in $36 \%$ of cases, $p<0.05$ ). ${ }^{7}$ It seems that corticosteroids give a more rapid improvement and less glandular ulceration into the airways than conventional treatment, but the eventual outcome is similar.

If there is no improvement after 1 month of treatment with TB drugs and corticosteroids, these patients are subjected to bronchoscopy. Bronchoscopy is used to determine the degree of airway obstruction and indicate whether further intervention is required. During bronchoscopy endoscopic enucleation of glands that have ulcerated into the airway and the resulting granulation tissue and caseating material can be removed. If at bronchoscopy severe airway compression is diagnosed, computed tomography (CT) of the chest is indicated.

CT scan is also useful to determine the anatomical location and nature of mediastinal glands (Fig. 1). Typical ghost-like enhancement of the rims of the lymph glands is visualised after contrast administration. The largest group of lymph nodes as identified by CT scan is located in the subcarinal area (87\%), and the most common sites of airway compression are the left main bronchus (21\%), right main bronchus (14\%) and bronchus intermedius $(8 \%){ }^{8}$

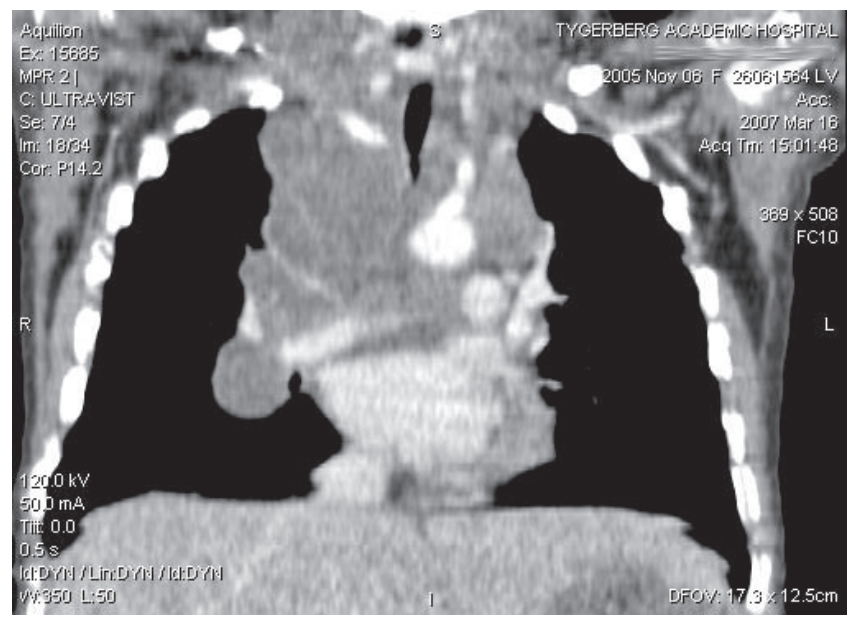

Fig. 1. A coronal reconstruction of a chest computed tomography scan demonstrating the large hilar, subcarinal and mediastinal tuberculosis lymph glands with compression of the trachea.

Surgical intervention in the management of complicated airway obstruction is only required for a small group of children. Clear indications for surgical enucleation are children requiring assisted ventilation for airway obstruction and children with life-threatening airway obstruction. Children with airway compression greater than $75 \%$ of the airway lumen, after 1 month of treatment to which corticosteroids have been added, should be considered for enucleation. Children with calcified lymph glands on CT scan are not good candidates as surgical enucleation will not be successful. The presence of lymph nodes alone is not an indication for surgery. Suggested indications include acute perforation of a major airway with severe respiratory embarrassment, pressure and occlusion of a major airway with lung collapse or hyperinflation, bronchial stenosis due to fibrosis and, rarely, superior vena cava obstruction or subcarinal oesophageal obstruction. ${ }^{9}$

The reported complication rate resulting from enucleation is low. Reported complications include bronchial tear, pulmonary artery laceration and bronchopleural fistula. 


\section{Original ARTICLES}

\section{Conclusion}

Most children with airway compression caused by TB lymph gland enlargement will respond favourably to medical treatment. A small percentage will need further investigation which includes bronchoscopy and chest CT scan. An even smaller percentage will benefit from surgical intervention.

\section{References}

Marais BJ, Gie RP, Schaaf HS, et al. The spectrum of disease in children treated for tuberculosis in a highly endemic area. Int J Tuberc Lung Dis 2006; 10: 1-7.

2. Theart A, Marais BJ, Gie RP, Hesseling AC, Beyers N. Criteria used for the diagnosis of childhood tuberculosis at primary health care level in a high-burden, urban setting. Int Tuberc Lung Dis 2005; 9: 1210-1214
3. Goussard P, Gie RP, Kling S, Beyers N. Expansile pneumonia in children caused by mycobacterium tuberculosis: clinical, radiological, and bronchoscopic appearances. Pediat Pulmonol 2004; 38: 451-455.

4. Fishman JE, Saraf-Lavi E, Narita M, et al. Pulmonary tuberculosis in AIDS patients; transient chest radiographic worsening after initiation of antiretroviral therapy. AJR 2000; 174: 43-49.

5. De Villiers RVP, Andronikou S, van der Westhuizen S. Specificity and sensitivity of chest radiographs in the diagnosis of paediatric pulmonary tuberculosis and the value of additional high-volt radiographs. Australas Radiol 2004; 48: 148-153.

6. Nemir RL, Cordona J, Lacoius A, David M. Prednisone as an adjunct in the chemotherapy of lymph node-bronchial tuberculosis in childhood: a double-blind study. Am Rev Respir Dis 1963; 88: 189-198.

7. Nemir RL, Cordona J, Vaziri F, Toledo F. Prednisone as an adjunct in the chemotherapy of lymph node-bronchial tuberculosis in childhood: a double-blind study. II. Further term observation. Am Rev Respir Dis 1967; 95: 402-410.

8. Andronikou S, Joseph E, Lucas S, et al. CT scanning for the detection of tuberculous mediastinal and hilar lymphadenopathy in children. Pediatr Radiol 2004; 34: 232-236.

9. Konstantinos A, Papagiannopoulos KA, Linegar AG, Harris DG, Rossouw GJ. Surgical management of airway obstruction in primary tuberculosis in children. Ann Thorac Surg 1999; 68: 1182-1186 